\title{
First-order Bogomol'nyī vortices on a sphere
}

\author{
Nuno M. Romão*† \\ DAMTP, University of Cambridge, Wilberforce Road, Cambridge CB3 0WA, UK \\ E-mail: 'nromao@mis.mpg.de',
}

\begin{abstract}
We describe the geometric quantisation of a nonrelativistic model of vortex dynamics introduced by Manton. If the vortices are placed on a sphere, the classical phase space is the compact manifold $\mathbb{C P}^{N}$ with a particular Kähler structure, and its symplectic volume can be related to the dimension of the quantum Hilbert space in a classical limit. Quantum operators corresponding to spatial isometries can be constructed explicitly and they allow for an interpretation of the solitons as interacting bosons.
\end{abstract}

\section{Introduction}

The diversity of field theories in $1+2$ dimensions has been increased in recent years by exploring the possibilities of the Chern-Simons action. Pure Chern-Simons gauge theory, although still interesting both from the physical and the mathematical points of view, has no dynamics by itself. However, many interesting models for field dynamics can be obtained by adding to the Chern-Simons action Maxwell or Yang-Mills terms and/or interactions with other fields [3i]. Some of these models have been shown to admit classical solitonic solutions (vortices), at least for critical or "self-dual" values of the parameters in the lagrangian. These objects can be regarded as smeared-out particles which retain a characteristic size and superpose nonlinearly; unlike some types of solitons, they can also be assigned a point-like core individually. In specific models, vortices often turn out to possess rather exotic properties, which may be relevant in applications. For example, models with abelian vortices have been important in attempts to explain phenomena in condensed matter theory such as superconductivity and the fractional quantum Hall effect.

In reference [1] $\left.\overline{1}_{1}^{\prime}\right]$, Manton constructed a nonrelativistic lagrangian for a $U(1)$ gauge field minimally coupled to a complex scalar on the plane which describes vortex dynamics. The action for the gauge field includes a Chern-Simons term and the purely spatial part of the Maxwell action. The equations of motion for the field theory are first-order in

\footnotetext{
${ }^{*}$ Speaker.

${ }^{\dagger}$ Current address: Max-Planck-Institut für Mathematik in den Naturwissenschaften, Inselstraße 22, D-04103 Leipzig, Germany
} 
time, and they admit the well-known Bogomol'ny vortices [i] $\left.{ }_{1}^{1} \overline{0}\right]$ of the Ginzburg-Landau theory as static solutions, for special values of the parameters. Mathematically, Bogomol'ny $\breve{1}$ vortices are rather well understood, even though on general surfaces they cannot be constructed analytically. Their space of gauge equivalence classes splits into disjoint sectors $\mathcal{M}_{N}$ labelled by an integer vortex number $N \in \mathbb{Z}$, each $\mathcal{M}_{N}$ (the moduli space of $N$ vortices) being a smooth $2|N|$-dimensional manifold. In his paper, Manton explored the dynamics of time-dependent fields by explicitly reducing the field theory lagrangian to an effective (finite-dimensional) mechanical system on the moduli space $\mathcal{M}_{N}$. The lagrangian equations of motion for the reduced system are again first-order in time, and so the moduli space is to be regarded as the phase space where a noncanonical hamiltonian dynamics takes place. The symplectic form defining the dynamics, determined by the kinetic term, contains nontrivial information about the system; of course, it may still be written in canonical form locally, but not in a natural way. Time evolution is determined by the potential energy alone, which is supposed to be small so that the field configurations are still approximately Bogomol'nyı̆ vortices. In the case of two vortices, the reduced system describes a rigid uniform rotation of the two vortex cores about their midpoint, with an angular velocity which only depends on the distance between the two cores.

If we place the vortices on a compact surface $\Sigma$, with a riemannian metric and an orientation, rather than on the plane, the moduli spaces $\mathcal{M}_{N}$ also become compact. The metric on $\Sigma$ fixes a complex structure, which in turn induces a complex structure on $\mathcal{M}_{N}$. This complex structure can be shown to be compatible with the symplectic form relevant for the dynamics, and so each $\mathcal{M}_{N}$ becomes a Kähler manifold. Compact Kähler

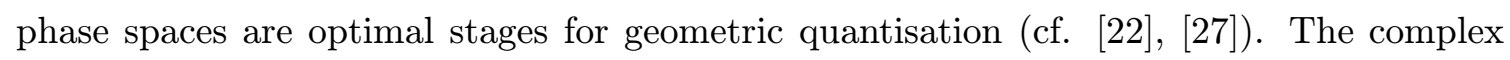
geometry supplies a natural (Kähler) polarisation, for which the corresponding quantum Hilbert space turns out to be finite-dimensional. This approach to the quantisation of the vortex system is to be included in a more general framework, pioneered in [i5i in the context of BPS monopoles. The idea is to probe the quantum behaviour of solitons through (geometric) quantisation of the reduced dynamics on the moduli space of static solutions, when such a space is available. In the more familiar situation of the abelian Higgs model $[\overline{2} \overline{0} \overline{0}]$, where the reduced system is second-order in time, there is a canonical hamiltonian description of the classical dynamics and the quantisation can be carried out using the vertical polarisation of $T^{*} \mathcal{M}_{N}$, which leads to a truncated Schrödinger representation of the quantum system. The accuracy of the approximation involved is very difficult to assess, and the study of an example where the Schrödinger representation is not available, as is the case here, is of considerable interest. From the point of view of geometric quantisation itself, it is fortunate that Manton's system seems to provide us with a nontrivial example where it may be put to work rather directly.

Our main aim is to discuss the geometric quantisation of Manton's reduced system of vortex dynamics, when space is taken to be a sphere of a given radius $R$. In section we describe Manton's model of vortex dynamics and summarise some results about the reduction to finite-dimensional mechanics obtained using the adiabatic approximation. In section $\overline{3}_{1}$, the setup for the geometric quantisation of the reduced dynamics on the moduli space of static solutions is presented. The dimension of the Hilbert space of wavefunctions 
is computed in section 'A Then we show how to construct the quantum angular momentum operators corresponding to the natural action of $S O(3)$ on the moduli space in section This allows some statements about the ground state of the dynamical regime $\lambda \neq 1$ to be made, which we discuss in section $\overline{6}_{1}^{\prime}$. We finish with some general comments about our results in section article [i] $[\overline{1} \bar{i}$ in].

\section{First-order dynamics of Bogomol'nyı̌ vortices}

Bogomol'nyı̌ vortices on a surface $\Sigma$ with a metric

$$
d s^{2}=\Omega^{2}(z, \bar{z}) d z d \bar{z}
$$

are gauge-equivalence classes of solutions $(A, \phi)$ to the equations

$$
\begin{gathered}
D_{\bar{z}} \phi=0, \\
2 B=\left(1-|\phi|^{2}\right) \Omega^{2},
\end{gathered}
$$

where we write $D=d-i A, D_{\bar{z}}=D_{\partial_{\bar{z}}}$ and $B=2 i\left(\partial_{\bar{z}} A_{z}-\partial_{z} A_{\bar{z}}\right)$. The gauge field $A$ is a connection 1-form for a $U(1)$ bundle $P$ over $\Sigma$ and $B$ is the curvature of this connection, whereas $\phi$ (the Higgs field) is a section of a line bundle associated to $P$. Topology is introduced on the fields through boundary conditions or, if $\Sigma$ is compact (which is the case we will be interested in), by the nontriviality of $P$. Topologically, $U(1)$ bundles on a compact surface are classified by their first Chern class $N \in \mathbb{Z}$, which can be interpreted as the net number $N \in \mathbb{Z}$ of units of quantised magnetic flux through space,

$$
\frac{i}{2} \oint_{\Sigma} B d z \wedge d \bar{z}=2 \pi N
$$

It can be shown that $N \geq 0$ for solutions to $(2 . \overline{2})$ and $(\overline{2} \cdot \overline{2} \cdot 3)$, and then $\phi$ vanishes at exactly $N$ points (counting multiplicities); similar equations describe configurations with $N<0$, called antivortices, but for simplicity we shall consider the case $N>0$ only.

Existence and uniqueness of Bogomol'nyı̆ vortices on compact $\Sigma$ were studied by Brad-

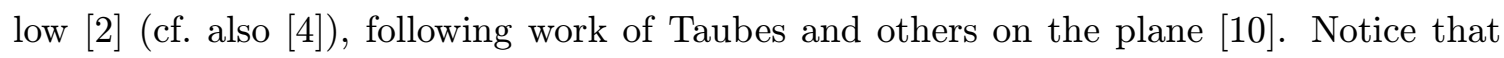
integration of both sides of equation ( $\left(\overline{2} \cdot \overline{3}^{\prime}\right)$ leads to

$$
\operatorname{Vol}(\Sigma) \geq 4 \pi N
$$

Bradlow showed that, whenever the strict inequality is satisfied, there is exactly one solution to the equation

$$
4 \partial_{z} \partial_{\bar{z}} h-\left(e^{h}-1\right) \Omega^{2}=4 \pi \sum_{r=1}^{N} \delta^{(2)}\left(z-z_{r}\right)
$$

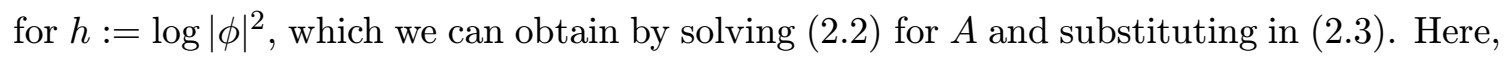
the $z_{r}$ are $N$ arbitrary points of $\Sigma$ where $\phi$ vanishes. Now $h$ contains all the gauge-invariant 
information about field configurations $(A, \phi)$, and so the moduli space of solutions to the Bogomol'nyı̆ equations with charge $N$ is is the symmetrised power $\mathcal{M}_{N}=S^{N} \Sigma:=\Sigma^{N} / \mathfrak{S}_{N}$, a smooth $2 N$-manifold. These solutions can be interpreted as nonlinear superpositions of $N$ indistinguishable vortices located at the zeros $z_{1}, \ldots, z_{N}$ of the Higgs field (the vortex cores), which play the rôle of moduli.

One can show that, in each topological sector, defined by $\left(\overline{2}_{2}^{-} \cdot \overline{4}^{\prime}\right)$, Bogomol'ny are exactly the minima of the Ginzburg-Landau energy $V=\int_{\Sigma} \mathcal{V}$, with density

$$
\mathcal{V}=\frac{1}{2} B^{2} \Omega^{-2}+\left(\left|D_{z} \phi\right|^{2}+\left|D_{\bar{z}} \phi\right|^{2}\right)+\frac{\lambda}{8}\left(1-|\phi|^{2}\right)^{2} \Omega^{2}
$$

at the self-dual point $\lambda=1$ [i] $\left[\begin{array}{l}1 \\ 1\end{array}\right]$ The functional $V$ is a natural potential energy for a dynamical field theory. Completing it so as to obtain a Lorentz-invariant action for $\Sigma=\mathbb{R}^{2}$ leads to the familiar abelian Higgs model, which is second-order (in the time derivatives of the fields) and can be defined on more general $\Sigma$. Another possibility, proposed by Manton

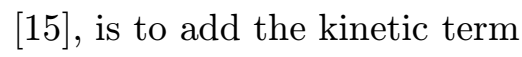

$$
\mathcal{T}=\gamma\left(\frac{i}{2}\left(\bar{\phi} D_{t} \phi-\phi \overline{D_{t} \phi}\right)-A_{t}\right) \Omega^{2}+\mu\left(B A_{t}+2 i\left(E_{z} A_{\bar{z}}-E_{\bar{z}} A_{z}\right)\right)
$$

where $E$ is defined from $d A=E \wedge d t+B$ in $1+2$ dimensions as usual. This leads to a firstorder lagrangian density $\mathcal{L}_{\text {Man }}=\mathcal{T}-\mathcal{V}$ for a nonrelativistic field theory. Notice that the second term in (2.8) is just the Chern-Simons action $\mu A \wedge d A$. There are some subtleties in defining an action globally from $\mathcal{L}_{\text {Man }}$, because some of the terms in $\mathcal{T}$ depend on the local gauge and cannot be patched together using partitions of unity. The action should also be gauge-invariant modulo $2 \pi \mathbb{Z}$. To deal with these difficulties, one may have to add correction boundary terms to the bulk action, restrict the group of large gauge transformations and/or impose quantisation conditions on the parameters in the lagrangian. For example, for compact $\Sigma$ and time-periodic boundary conditions, one is led to a well-defined action consistent with all large gauge transformations provided we impose [i] $[\overline{1} \overline{8}]$

$$
4 \pi \mu \in \mathbb{Z} \quad \text { and } \quad \gamma \operatorname{Vol}(\Sigma) \in \mathbb{Z} .
$$

The equations of motion are gauge-invariant and satisfied by time-independent Bogomol'nyı vortices provided $\gamma=\mu$, which we will assume from now on. In particular, (2.2.3) coincides with the constraint obtained as the Euler-Lagrange equation for the lagrange multiplier $A_{t}$. It is useful to define the normalised coefficient $\kappa:=4 \pi \mu=4 \pi \gamma$.

From now on, we shall focus on the special case where $\Sigma$ is a sphere of radius $R$. We take $z$ to be the complex coordinate obtained through stereographic projection from the South pole of a unit sphere, and set

$$
\Omega^{2}(z, \bar{z})=\frac{4 R^{2}}{\left(1+|z|^{2}\right)^{2}}
$$

in $(2 . \overline{1})$. The positions $z_{1}, \ldots, z_{N}$ of $N$ vortices define coordinates almost everywhere on the moduli space $\mathcal{M}_{N}$. They are regular only in the subset of configurations for which all the zeros of $\phi$ are simple and none of them occur at the South pole, which we denote as 
usual by $z=\infty$. To parametrise the whole subset $V_{(0)} \subset \mathcal{M}_{N}$ of static configurations with no zeros of the Higgs field at $z=\infty$, we can introduce instead the elementary symmetric polynomials in the $N$ variables $z_{r}$ :

$$
s_{k}:=s_{k}^{[N]}\left(z_{1}, \ldots, z_{N}\right)=\sum_{1 \leq j_{1}<\cdots<j_{k} \leq N} z_{j_{1}} \cdots z_{j_{k}}, \quad 1 \leq k \leq N .
$$

Stereographic projection from another point of the sphere would give another complex coordinate $z^{\prime}$ related to $z$ by a Möbius transformation (representing a rotation), and then a coordinate patch $V_{\left(0^{\prime}\right)}$ on $\mathcal{M}_{N}$ with coordinates $s_{k}^{\prime}$. An atlas for the whole moduli space can be construced by projecting from $N+1$ distinct points. To see what the gobal structure of $\mathcal{M}_{N}$ is, let $V_{j}$ denote the subset of configurations with exactly $j$ vortices at $z=\infty$; it is parametrised by the symmetric polynomials $s_{k}^{[N-j]}$ of the coordinates of the $N-j$ remaining vortices. Clearly, $\mathcal{M}_{N}=\coprod_{j=0}^{N} V_{j}$ gives a decomposition of $\mathcal{M}_{N}$ into $N+1$ disjoint $2(N-j)$-cells $V_{j} \cong \mathbb{C}^{N-j}$, and it is easy to verify that they are glued together so as to give $\mathcal{M}_{N}=\mathbb{C P}^{N}$.

We are interested in the dynamics of the vortices in the field theory, and this is a very hard problem. One way of making progress, in the $\lambda \simeq 1$ regime, is to obtain an effective finite-dimensional lagrangian system on the moduli space by substituting Bogomol'nyı vortices with time-dependent moduli for the fields in the action and integrating out the spatial dependence; this is the so-called adiabatic approximation, first discussed by Manton in the context of BPS monopoles [i] ${ }_{1}^{1} \overline{3}$. It turns out that the integral for the kinetic energy can be performed explicitly. The result can be written as

$$
T^{\mathrm{red}}=\mathcal{A}\left(\frac{d}{d t}\right),
$$

where the real 1-form

$$
\mathcal{A}=\pi i \gamma \sum_{r=1}^{N}\left[\left(2 R^{2} \frac{\bar{z}_{r}}{1+\left|z_{r}\right|^{2}}+\tilde{b}_{r}\right) d z_{r}-\left(2 R^{2} \frac{z_{r}}{1+\left|z_{r}\right|^{2}}+\overline{\tilde{b}}_{r}\right) d \bar{z}_{r}\right]
$$

depends on $N$ functions $\tilde{b}_{r}$ on $V_{(0)}$. To define them, suppose first that $z_{1}, \ldots, z_{N}$ are

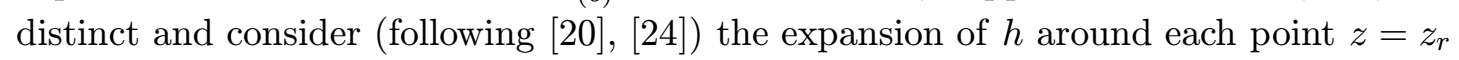

$$
h\left(z ; z_{1}, \ldots, z_{N}\right)=\log \left|z-z_{r}\right|^{2}+a_{r}+\frac{b_{r}}{2}\left(z-z_{r}\right)+\frac{\bar{b}_{r}}{2}\left(\bar{z}-\bar{z}_{r}\right)+\mathcal{O}\left(\left|z-z_{r}\right|^{2}\right) .
$$

Equation $(2.6)$ implies that the $b_{r}$ have poles as the vortices coalesce, in such a way that

$$
\tilde{b}_{r}\left(z_{1}, \ldots, z_{N}\right):=b_{r}\left(z_{1}, \ldots, z_{N}\right)-\sum_{s \neq r}^{N} \frac{2}{z_{r}-z_{s}}
$$

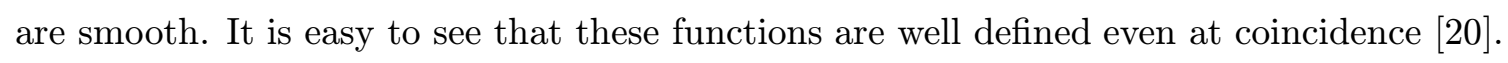
It also follows from (2. $\left(\bar{b}^{2}\right)$ that, under an isometry $T$, the $b_{r}$ transform as

$$
T^{*} b_{r}=\frac{b_{r}}{T^{\prime}\left(z_{r}\right)}-\frac{T^{\prime \prime}\left(z_{r}\right)}{\left(T^{\prime}\left(z_{r}\right)\right)^{2}}
$$


and this can be used to relate $b_{r}$ (or $\tilde{b}_{r}$ ) in the patch $V_{(0)}$ to the $b_{r}^{\prime}$ defined in another patch $V_{\left(0^{\prime}\right)}$ from an expansion like (2.12).

Introducing $\omega:=d \mathcal{A}$, the equations of motion can be cast as

$$
\iota_{\frac{d}{d t}} \omega=-d V^{\mathrm{red}}
$$

with

$$
V^{\mathrm{red}}=N \pi+\frac{i}{16}(\lambda-1) \int_{\Sigma}\left(1-e^{h\left(z ; z_{1}, \ldots, z_{N}\right)}\right)^{2} \Omega^{2} d z \wedge d \bar{z} .
$$

So the reduced dynamics is hamiltonian, with a nontrivial symplectic structure defined by $\omega$, and hamiltonian given by the potential energy $V^{\text {red }}$. (Unfortunately, it does not seem possible to write down $V^{\text {red }}$ as a local function on $\mathcal{M}_{N}$.) It follows form the work of Samols $[\overline{2} \overline{0} \overline{0}]$ that there is a real function $\mathcal{B}$ on $V_{(0)}$ such that

$$
\tilde{b}_{r}=\frac{\partial \mathcal{B}}{\partial z_{r}}
$$

and using this we find

$$
\omega=-\frac{i \kappa}{2} \sum_{r, s=1}^{N}\left(\frac{2 R^{2} \delta_{r s}}{\left(1+\left|z_{r}\right|^{2}\right)^{2}}+\frac{\partial \bar{b}_{s}}{\partial z_{r}}\right) d z_{r} \wedge d \bar{z}_{s} .
$$

We can recognise $\omega_{\text {Sam }}=-\frac{2}{\kappa} \omega$ as the $(1,1)$-form defining the Kähler metric on $\mathcal{M}_{N}$ whose

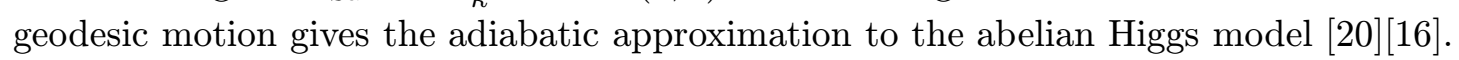

In [i' $\left.\overline{1} \bar{z}_{1}\right]$, we analysed in some detail the symmetries of Manton's model. Here, we shall focus on the reduced picture. The fact that $\mathcal{V}$ in $(\overline{2} . \overline{1})$ does not depend on time explicitly means that it is a conserved quantity (the energy of the system). There are also conserved angular momenta coming from the rotational symmetry, which is described infinitesimally by the span of the vector fields

$$
\begin{aligned}
& \xi_{(1)}=-\frac{i}{2} \sum_{r=1}^{N}\left(\left(1-z_{r}^{2}\right) \frac{\partial}{\partial z_{r}}-\left(1-\bar{z}_{r}^{2}\right) \frac{\partial}{\partial \bar{z}_{r}}\right) \\
& \xi_{(2)}=\frac{1}{2} \sum_{r=1}^{N}\left(\left(1+z_{r}^{2}\right) \frac{\partial}{\partial z_{r}}+\left(1+\bar{z}_{r}^{2}\right) \frac{\partial}{\partial \bar{z}_{r}}\right) \\
& \xi_{(3)}=i \sum_{r=1}^{N}\left(z_{r} \frac{\partial}{\partial z_{r}}-\bar{z}_{r} \frac{\partial}{\partial \bar{z}_{r}}\right)
\end{aligned}
$$

which generate rotations around the three cartesian axes of an $\mathbb{R}^{3}$ where the sphere $\Sigma$ is embedded. Equation ( $(2.13 i)$ can be used to derive constraints on the functions $b_{r}\left[\begin{array}{l}1 \\ 1\end{array} \overline{8} \overline{1}\right]$,

$$
\begin{gathered}
\sum_{s=1}^{N}\left(2 z_{s}+z_{s}^{2} b_{s}+\bar{b}_{s}\right)=0, \\
\sum_{s=1}^{N} z_{s} b_{s} \in \mathbb{R}
\end{gathered}
$$


which imply that the $S O(3)$ action induced on $\mathcal{M}_{N}$ is symplectic. Since $\mathfrak{s o}(3)$ is simple, the action is necessarily hamiltonian, and there exists a moment map [i];]

$$
\mathbf{M}: \mathcal{M}_{N} \rightarrow \mathfrak{s o}(3)^{*}
$$

with components defined by hamiltonian functions $M_{j}$ of $(2.18 \mathrm{i})-\left(2 . \overline{2} 0^{\circ}\right)$,

$$
\iota_{\xi_{(j)}} \omega=-d M_{j}
$$

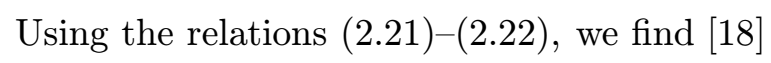

$$
\begin{aligned}
& M_{1}= \pm \frac{\kappa}{4} \sum_{r=1}^{N}\left(2 R^{2} \frac{z_{r}+\bar{z}_{r}}{1 \pm\left|z_{r}\right|^{2}}+b_{r}+\bar{b}_{r}\right), \\
& M_{2}=\mp \frac{i \kappa}{4} \sum_{r=1}^{N}\left(2 R^{2} \frac{z_{r}-\bar{z}_{r}}{1 \pm\left|z_{r}\right|^{2}}-\left(b_{r}-\bar{b}_{r}\right)\right), \\
& M_{3}= \pm \frac{\kappa}{2} \sum_{r=1}^{N}\left(R^{2} \frac{1 \mp\left|z_{r}\right|^{2}}{1 \pm\left|z_{r}\right|^{2}} \mp\left(z_{r} b_{r}+1\right)\right) .
\end{aligned}
$$

These conserved quantities can be compared in the field theory and in the reduced mechanical system, providing some evidence for the consistency of the adiabatic approximation as applied to this model. They are natural classical observables, and we shall be able to find quantum operators for them in closed form.

\section{Ingredients for geometric quantisation}

Now we would like to investigate the quantum system associated to the reduced Manton's model in the framework of geometric quantisation. We shall follow the conventions in

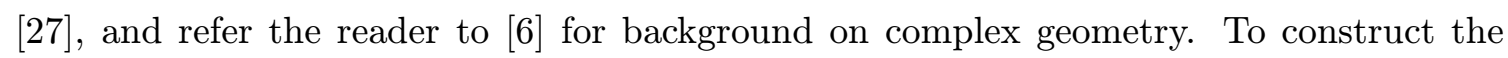
quantum system, we need to supplement the classical theory (specified by the phase space $\mathcal{M}_{N}=\mathbb{C P}^{N}$, endowed with the symplectic form (2.17)) with a hermitian line bundle $L$ over $\mathcal{M}_{N}$. The wavefunctions in the quantum Hilbert space will be particular sections of $L$.

To start with, we should verify whether our phase space is quantisable at all. This is equivalent to the integrality of the class represented by the closed form $\frac{1}{2 \pi} \omega$ in de Rham cohomology,

$$
\frac{1}{2 \pi}[\omega] \in H^{2}\left(\mathcal{M}_{N} ; \mathbb{Z}\right) \subset H^{2}\left(\mathcal{M}_{N} ; \mathbb{R}\right) .
$$

In general, this requirement leads to nontrivial constraints on the parameters of the classical theory - the Weil (pre)quantisation conditions. If they are satisfied, we may regard $\frac{1}{2 \pi}[\omega]$ as the first Chern class of a smooth complex line bundle over $\mathcal{M}_{N}$, which is what we call the prequantum line bundle $L$.

Recall that $H^{2}\left(\mathbb{C P}^{N} ; \mathbb{R}\right)$ is cyclic and we can take as generator the first Chern class $\eta \in H^{2}\left(\mathbb{C P}^{N} ; \mathbb{Z}\right)$ of the hyperplane bundle of $\mathbb{C P}^{N}$. Then $[\omega]=2 \pi \ell \eta$ for suitable $\ell \in \mathbb{R}$. To 
obtain $\ell$, we can use the result for the cohomology class $\left[\omega_{\text {Sam }}\right]$ derived by Manton in [i] 1 ini $]$; it involves obtaining an explicit formula for the restriction of the 2-form $\omega$ to the subvariety $\mathcal{M}_{N}^{\text {co }}$ of coincident $N$-vortices, which can be seen to be a projective line of degree $N$ inside $\mathcal{M}_{N}=\mathbb{C P}^{N}$. (This formula has been generalised in [1] $\overline{1}_{1}^{1}$ for $\Sigma$ of arbitrary genus using similar arguments.) The conclusion is that that $\ell=-\kappa\left(R^{2}-N\right)$, or

$$
\frac{1}{2 \pi}[\omega]=\ell \eta=-\kappa\left(R^{2}-N\right) \eta
$$

Equation (3.

$$
\kappa\left(R^{2}-N\right) \in \mathbb{Z}
$$

Notice that this condition is weaker than the conditions $(2.9)$ that we would have to be impose at the classical level if we were to allow gauge transformations to have nontrivial winding in the time circle when periodic boundary conditions are enforced. On the other hand, if we choose to restrict the group of gauge transformations to small gauge transformations, $\left(\overline{2} . \bar{y}_{1}\right)$ are no longer required and $(\overline{3} . \overline{3})$ is a nontrivial extra condition on the parameters for the quantum mechanics to make sense.

In geometric quantisation, the prequantum line bundle $L$ is to be equipped with a hermitian metric and a unitary connection. The fact that $\mathbb{C P}^{N}$ is simply connected implies that in our case $L$ is uniquely determined as a smooth bundle by the symplectic structure $\omega$, and so are the hermitian metric and the connection $\mathcal{D}$. The basic idea in the standard construction of $L$ is to interpret (real) symplectic potentials of $\omega$ as local expressions for the connection, and then use a version of parallel transport with path-labels to define local sections and construct the bundle. (This works even if the connection is not flat, cf. section 8.3 of $[\overline{2} \overline{2} \overline{-}]$.) A given symplectic potential determines a unique local section $\sigma$ of $L$ up to a phase of modulus one. The hermitian metric is introduced by requiring that each $\sigma$ is a local orthonormal frame,

$$
\langle\sigma, \sigma\rangle=1
$$

This is unambiguous since two symplectic potentials must differ by the exterior derivative of a real function $u$, and then the corresponding local sections are related by the factor $e^{-i u}$.

The wavefunctions in geometric quantisation are defined as the $L^{2}$ polarised sections of $L$. By $L^{2}$ we mean square-integrable with respect to the hermitian product (3.4. $)$ on the fibres and the symplectic measure $\frac{\omega^{N}}{N !}$ on the base $\mathcal{M}_{N}$. Roughly speaking, polarised means that they only depend on half of the real coordinates of the phase space, just as the wavefunctions in the Schrödinger representation of quantum mechanics only depend on the position and not on the momentum. More precisely, a polarisation $\mathcal{P}$ is defined as a lagrangian (i.e. maximally isotropic) integrable subbundle of the complexification $T_{\mathbb{C}} \mathcal{M}_{N}$ of the tangent bundle of the phase space, and the condition

$$
\mathcal{D}_{\bar{X}} \psi=0, \quad \forall X \in \Gamma\left(\mathcal{M}_{N}, \mathcal{P}\right)
$$


defines what is meant for a section $\psi$ to be $\mathcal{P}$-polarised. When the classical dynamics takes place in a Kähler phase space, as is our case, there is a natural choice of polarisation $\mathcal{P}$ - namely, the one determined by the $i$-eigenspaces of the compatible complex structure. It is generated by the holomorphic vector fields in the local complex coordinates. The introduction of a Kähler polarisation can be interpreted naturally in terms of complex geometry as follows. A connection on the prequantum line bundle defines a holomorphic structure for $L$ : By definition, the holomorphic sections are the ones which are annihilated by the part of $\mathcal{D}$ that takes values in $\Omega^{(0,1)}\left(\mathcal{M}_{N}, L\right)$, which is defined from the complex structure on the base. (The existence of such sections relies on the fact that the Kähler form has no $(0,2)$-component.) But these sections are precisely the ones satisfying the condition (3. holomorphic sections with respect to the holomorphic structure on $L$ induced by the unitary connection $\mathcal{D}$.

\section{The quantum Hilbert space}

In algebraic geometry, isomorphism classes of line bundles of degree zero over an algebraic variety are classified by an abelian group (under the tensor product) called the Picard variety. This is just the trivial group when the base is $\mathbb{C P}^{N}$, which implies that in our problem $L$ is uniquely determined as a holomorphic line bundle by its first Chern class $\ell$,

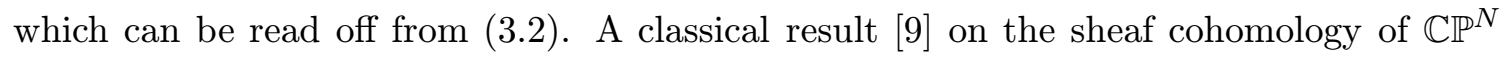
establishes that $L \cong \mathcal{O}(\ell)$ admits global holomorphic sections if and only if $\ell>0$ (i.e. $\kappa<0)$, and then they form the vector space

$$
H^{0}\left(\mathbb{C P}^{N}, \mathcal{O}(\ell)\right) \cong \mathbb{C}\left[Y_{0}, \ldots, Y_{N}\right]_{\ell}
$$

where the right-hand side denotes the homogeneous polynomials of degree $\ell$ in $N+1$ homogeneous coordinates $Y_{j}$ for $\mathbb{C P}^{N}$. We fix the coordinates $Y_{j}$ by identifying $\frac{Y_{j}}{Y_{0}}=$ $(-1)^{r} s_{r}(r=1, \ldots, N)$ on $\left\{\left(Y_{0}: \cdots: Y_{N}\right) \in \mathbb{C P}^{N}: Y_{0} \neq 0\right\}=V_{(0)}$, where the $s_{r}=s_{(0) r}$ are given by $(2,100)$. The isomorphism $\left(\overline{1}_{1}^{4} \cdot \overline{1}_{1}^{\prime}\right)$ gives a concrete way to realise $L$ and its holomorphic sections, up to multiplication by a constant in $\mathbb{C}^{*}$.

Recall that the local symplectic potential $\mathcal{A}$ in $(2,1 \overline{1})$ for the connection $\mathcal{D}$ determines a nonvanishing local section $\sigma: V_{(0)} \rightarrow L$. It is not holomorphic though, as $\mathcal{A}$ has a nonzero component in $\Omega^{(0,1)}\left(V_{(0)}\right)$. But we can obtain a holomorphic local section from it by using a nonunitary gauge transformation: Since

$$
\mathcal{A}=2 \pi \gamma i \sum_{r=1}^{N}\left(2 R^{2} \frac{\bar{z}_{r}}{1+\left|z_{r}\right|^{2}}+\tilde{b}_{r}\right) d z_{r}-2 \pi \gamma i d\left(\frac{1}{2} \mathcal{B}+R^{2} \sum_{r=1}^{N} \log \left(1+\left|z_{r}\right|^{2}\right)\right),
$$

where $\mathcal{B}$ is defined up to an additive real constant by $(2 . \overline{1})$, we can define on $V_{(0)}$

$$
\sigma^{(0)}\left(z_{1} \ldots, z_{N}\right):=\sigma\left(z_{1} \ldots, z_{N}\right) e^{-2 \pi \gamma\left(\frac{1}{2} \mathcal{B}+R^{2} \sum_{r=1}^{N} \log \left(1+\left|z_{r}\right|^{2}\right)\right)}
$$

this is a holomorphic section of $L$ on $V_{(0)}$. It is uniquely determined from $\sigma$ up to a positive real constant, and thus from $\mathcal{A}$ up to a constant in $\mathbb{C}^{*}$. It extends to a global 
section of $L$, vanishing in the complement of $V_{(0)}$; so we identify it with the homogeneous

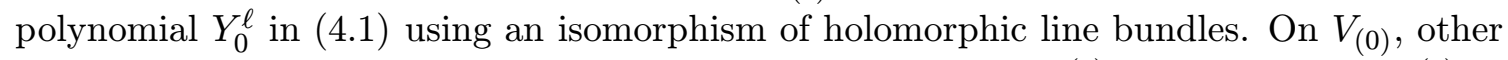
global holomorphic sections $\Psi$ of $L$ are given by multiplying $\sigma^{(0)}$ by polynomials $\Psi^{(0)}$ in the $s_{r}$ of degree less than or equal to $\ell$. It is possible to trivialise $L$ in other patches $V_{\left(0^{\prime}\right)}$ as suggested in section $\overline{2}$, and construct the transition functions describing the bundle (more details are given in $\left[\underline{1}_{i}^{i}\right)$; then we can find the representatives of the global holomorphic sections of $L$ and the connection 1-form in any patch.

The quantum Hilbert space $\mathcal{H}_{\mathcal{P}}$ is the space of global holomorphic sections of $L$ which are normalisable with respect to the inner product defined by the symplectic measure of $\mathcal{M}_{N}$ and the product on the fibres given by $(\overline{3}-4)$, as we already explained in section This inner product can be easily written down as an integral over the open dense $V_{(0)}$,

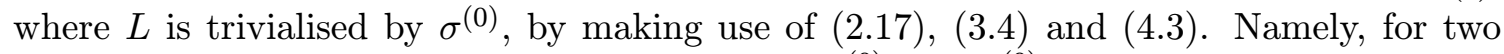
holomorphic sections $\Psi_{1}$ and $\Psi_{2}$ represented by $\Psi_{1}^{(0)}$ and $\Psi_{2}^{(0)}$ with respect to $\sigma^{(0)}$,

$$
\begin{aligned}
\left\langle\Psi_{1}, \Psi_{2}\right\rangle= & \left(\frac{i \kappa}{2}\right)^{N} \int_{\mathbb{C}^{N}} \overline{\Psi_{1}^{(0)}\left(z_{1}, \ldots, z_{N}\right)} \Psi_{2}^{(0)}\left(z_{1}, \ldots, z_{N}\right) e^{-4 \pi \gamma\left(\frac{1}{2} \mathcal{B}+R^{2} \sum_{p=1}^{N} \log \left(1+\left|z_{p}\right|^{2}\right)\right)} \\
& \times \operatorname{det}\left[\frac{2 R^{2} \delta_{r s}}{\left(1+\left|z_{r}\right|^{2}\right)^{2}}+\frac{\partial \bar{b}_{s}}{\partial z_{r}}\right]_{r, s=1}^{N} d z_{1} \wedge d \bar{z}_{1} \wedge \ldots \wedge d z_{N} \wedge d \bar{z}_{N}
\end{aligned}
$$

Since we are dealing with a compact phase space, all the holomorphic sections have finite norm, so the Hilbert space $\mathcal{H}_{\mathcal{P}}$ is $H^{0}\left(\mathbb{C P}^{N}, \mathcal{O}(L)\right)$ itself, with dimension

$$
\operatorname{dim} \mathcal{H}_{\mathcal{P}}=\left(\begin{array}{c}
N+\ell \\
N
\end{array}\right) .
$$

We should mention at this stage that the $\mathbb{C}^{*}$ ambiguity underlying the identification of the sections (4. ambiguity in the definition of the inner product ( $\left(\bar{L}^{-} \overline{4}^{\prime}\right)$. This is no shortcoming, since from the physical point of view the quantum states are points in $\mathbb{P}\left(\mathcal{H}_{\mathcal{P}}\right)$, and a global renormalisation of the representatives in $\mathcal{H}_{\mathcal{P}}$ has no physical implications.

We may interpret the expression ( system of $N$ interacting bosons. By interacting, we mean that the area available for the dynamics on the sphere is affected by the space which the vortices themselves occupy, in the

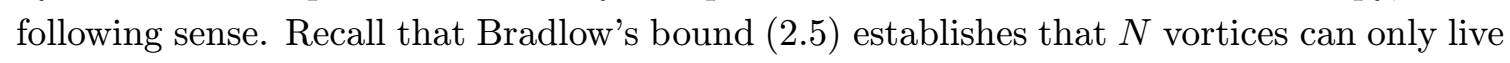
on a sphere which has an area exceeding $4 \pi N$. Heuristically, we can say that a single vortex occupies $4 \pi$ units of area. So we can regard (4. states for a system of $N$ bosons which can be assigned to any of the $\ell=\frac{|\kappa|}{4 \pi}\left(4 \pi R^{2}-4 \pi N\right)$ states corresponding to the room available on the sphere, after the total area of the vortices has been discounted. (For $\kappa=-1$, there is a similiar interpretation for (4.5) as the number of states of a system of $N$ noninteracting fermions, but it breaks down for $\kappa \neq-1$.) All these quantum states belong to a single degenerate energy level when $\lambda=1$. Recall that in this situation the hamiltonian vanishes and no motion occurs at the classical level. We shall refer to the $\lambda \neq 1$ case in section 'í, below. 
From the formula (3) determined by the Kähler form $\omega$ :

$$
\operatorname{Vol}_{\omega}\left(\mathcal{M}_{N}\right)=\frac{\left(2 \pi|\kappa|\left(R^{2}-N\right)\right)^{N}}{N !}=\frac{(2 \pi \ell)^{N}}{N !} .
$$

It is of course proportional to the volume determined by Samols' metric, as first computed

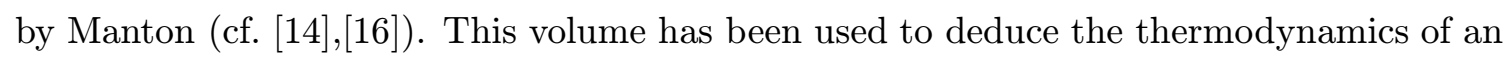
ideal gas of abelian Higgs vortices at $\lambda=1$ in the framework of Gibbs' classical statistical mechanics. In Manton's model at $\lambda=1$, there is only a ground state as we noted above, and its degeneracy, in Gibbs' approximation, is given by

$$
d_{\text {Gibbs }}=\frac{1}{(2 \pi \hbar)^{N}} \operatorname{Vol}\left(\mathcal{M}_{N}\right)=\frac{\ell^{N}}{N !} .
$$

Notice that Planck's constant is $2 \pi \hbar=2 \pi$ in our units. Gibbs' partition function is simply $Z_{\text {Gibbs }}=d_{\text {Gibbs }} e^{-\beta N \pi}$. At $\lambda \neq 1$, the degeneracy is lifted but (4. as the total number of states of the system. It is of interest to study the quotient

$$
Q:=\frac{\operatorname{dim} \mathcal{H}_{\mathcal{P}}}{d_{\text {Gibbs }}}
$$

which gives information about how appropriate Gibbs' estimate for the number of states

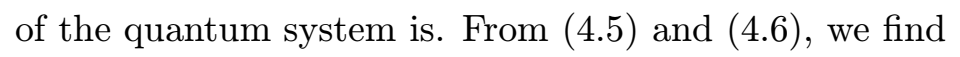

$$
Q=\frac{(N+\ell) !}{\ell^{N} \ell !}
$$

Using Stirling's formula for the gamma function, we obtain

$$
Q=\left(1+\frac{N+1}{\ell}\right)^{N}\left(1+\frac{N}{\ell+1}\right)^{-\frac{1}{2}}\left[\left(1+\frac{N}{\ell+1}\right)^{\ell+1} e^{-N}\right] e^{J(N+\ell+1)-J(\ell+1)},
$$

where $J$ is the asymptotic series

$$
J(z)=\sum_{n=1}^{\infty} \frac{B_{n}}{(2 n-1) 2 n} \frac{1}{z^{2 n-1}} .
$$

In the context of Chern-Simons theories, the classical approximation is described as the limit $|\kappa| \rightarrow \infty$; this is equivalent to keeping the coupling $\kappa$ as constant and letting $\hbar \rightarrow 0$. So we keep $N$ fixed and let $\ell \rightarrow \infty$ in the expression ( $\left.\overline{1}^{2} \overline{7}^{\prime}\right)$, and this gives indeed $Q \rightarrow 1$. We might also try to obtain a classical regime in a thermodynamical limit, where both $N$ and the area of the sphere become very large, but keeping a finite (possibly small) density, which we might want to define as

$$
\nu:=\frac{|\kappa| N}{\ell}=\frac{\frac{N}{R^{2}}}{\left(1-\frac{N}{R^{2}}\right)} .
$$

But it follows from ( $\left.\overline{4}^{-} \overline{7}_{1}\right)$ that in this limit $Q$ is infinite, however small $\nu$ is taken to be. We mention in passing that there is a physical realisation for Manton's lagrangian (see section ii'i) in which $|\kappa| R^{2} \rightarrow \infty$ is to be regarded as a large-number limit, and then $\lim _{\ell \rightarrow \infty} Q=1$ can alternatively be interpreted as the consistency of Gibbs' recipe in a thermodynamical regime. 


\section{Quantum angular momenta}

From the prequantisation data, it is possible to construct prequantum operators $\mathcal{Q}(f)$ for any classical observable $f \in C^{\infty}\left(\mathcal{M}_{N}\right)$ as

$$
\mathcal{Q}(f):=-i \mathcal{D}_{\xi_{f}}+f
$$

Here, $\xi_{f}$ is the hamiltonian vector field of $f$ with respect to $\omega$,

$$
\iota_{\xi_{f}} \omega=-d f
$$

$(2,14)$ and $(2.23)$ being special cases of this. In general, the linear operator $\mathcal{Q}(f)$ does not map polarised sections of $L$ to polarised sections. It is easy to show that it does if and only if $\xi_{f}$ preserves the polarisation:

$$
\left[\xi_{f}, \Gamma\left(\mathcal{M}_{N}, \mathcal{P}\right)\right] \subset \Gamma\left(\mathcal{M}_{N}, \mathcal{P}\right)
$$

Then we may interpret $\mathcal{Q}(f)$ as the quantum operator ${ }^{1}$ corresponding to the observable $f$. In the Kähler case, (1) can be seen to be equivalent to $\xi_{f}$ being the real part of a holomorphic vector field. This condition is true for the hamiltonian vector fields (2.18i)$\left(2.20_{1}^{\prime}\right)$ of the angular momenta in $(2.24)-(2.26)$.

We can determine explicitly the action of the quantum operators on the wavefunctions $\Psi$ in the quantum Hilbert space $\mathcal{H}_{\mathcal{P}}=H^{0}\left(\mathcal{M}_{N}, \mathcal{O}(L)\right)$. In the holomorphic frame on $V_{(0)}$ provided by $\sigma^{(0)}$, one can write $\Psi=\Psi^{(0)} \sigma^{(0)}$ with

$$
\Psi^{(0)}\left(z_{1}, \ldots, z_{N}\right)=\sum_{j_{1}+\cdots+j_{N}=0}^{\ell} \alpha_{j_{1} \ldots j_{N}} \prod_{k=1}^{N} s_{k}^{[N]}\left(z_{1}, \ldots, z_{N}\right)^{j_{k}}
$$

where $\alpha_{j_{1} \ldots j_{N}} \in \mathbb{C}$. The 1 -form representing $\mathcal{D}$ with respect to this frame can be read off

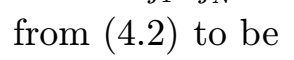

$$
\mathcal{A}^{(0)}=2 \pi \gamma i \sum_{r=1}^{N}\left(2 R^{2} \frac{\bar{z}_{r}}{1+\left|z_{r}\right|^{2}}+\tilde{b}_{r}\right) d z_{r}
$$

Substitution in $(5.1)$ now gives the local representatives of the quantum operators in the local frame $\sigma^{(0)}$. For example, for $M_{3}$ we obtain

$$
\begin{aligned}
\mathcal{Q}\left(M_{3}\right) & =-i\left(\iota_{\xi_{(3)}} d-i \mathcal{A}^{(0)}\left(\xi_{(3)}\right)\right)+M_{3} \\
& =-i \iota_{\xi_{(3)}} d+\frac{\kappa}{2} N\left(R^{2}-1\right)-\kappa \sum_{r=1}^{N} \sum_{s \neq r}^{N} \frac{z_{r}}{z_{r}-z_{s}} \\
& =-i \iota_{\xi_{(3)}} d-\frac{N \ell}{2},
\end{aligned}
$$

\footnotetext{
${ }^{1}$ For a general $f \in C^{\infty}\left(\mathcal{M}_{N}\right)$, the prequantum operator defined by $\left({ }^{2} \cdot \bar{l}_{1}^{1}\right)$ has to be corrected (e.g. using the BKS construction, cf. [2요 2 ) to obtain the (true) quantum operator $\mathcal{Q}(f)$.
} 
or equivalently

$$
\mathcal{Q}\left(M_{3}\right)=\sum_{r=1}^{N}\left(z_{r} \frac{\partial}{\partial z_{r}}-\bar{z}_{r} \frac{\partial}{\partial \bar{z}_{r}}\right)-\frac{N \ell}{2} .
$$

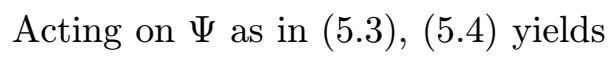

$$
\mathcal{Q}\left(M_{3}\right) \Psi^{(0)}=\sum_{j_{1}+\cdots+j_{N}=0}^{\ell}\left(j_{1}+2 j_{2}+\cdots+N j_{N}-\frac{N \ell}{2}\right) \alpha_{j_{1} \ldots j_{N}} \prod_{k=1}^{N} s_{k}^{[N]}\left(z_{1}, \ldots, z_{N}\right)^{j_{k}} .
$$

From this expression, it is easy to read off the eigenvalues of $\mathcal{Q}\left(M_{3}\right)$ as

$$
-\frac{N \ell}{2},-\frac{N \ell}{2}+1, \ldots, \frac{N \ell}{2}
$$

together with their multiplicities. The same spectrum is obtained for $\mathcal{Q}\left(M_{1}\right)$ and $\mathcal{Q}\left(M_{2}\right)$, with quantum operators given by

$$
\begin{aligned}
& \mathcal{Q}\left(M_{1}\right)=-\frac{1}{2} \sum_{r=1}^{N}\left(\left(1-z_{r}^{2}\right) \frac{\partial}{\partial z_{r}}-\left(1-\bar{z}_{r}^{2}\right) \frac{\partial}{\partial \bar{z}_{r}}\right)-\frac{\ell}{2} \sum_{r=1}^{N} z_{r} \\
& \mathcal{Q}\left(M_{2}\right)=-\frac{i}{2} \sum_{r=1}^{N}\left(\left(1+z_{r}^{2}\right) \frac{\partial}{\partial z_{r}}+\left(1+\bar{z}_{r}^{2}\right) \frac{\partial}{\partial \bar{z}_{r}}\right)+\frac{i \ell}{2} \sum_{r=1}^{N} z_{r}
\end{aligned}
$$

acting on polynomials of the form $\left(\overline{5}^{2} \cdot 3 i\right)$.

For $N=1$ and a given negative $\kappa \in \mathbb{Z}$, we see that the Hilbert space $\mathcal{H}_{\mathcal{P}}$ yields the irreducible (possibly projective) $(\ell+1)$-dimensional representation of $S O(3)$ through the action of the generators $\mathcal{Q}\left(M_{j}\right)$. The situation here is exactly equivalent to the geometric quantisation of the spin degrees of freedom of a particle of spin $\frac{\ell}{2}$, which are described classically by a two-sphere of half-integer radius $\frac{\ell}{2}$ and the standard Fubini-Study symplectic form [22]. More generally, for any $N$, it follows from $(5.5)$ that the representation of $S O(3)$ carried by $\mathcal{H}_{\mathcal{P}}$ is the $N$ th symmetric power $\operatorname{Sym}^{N}(\ell+\mathbf{1})$; notice that $\ell$ itself depends on $N$. The fact that we obtain a symmetric power of an irreducible representation with heighest weight determined by $\ell$ again indicates that the vortices in our model can be regarded as interacting bosons, as we have put forward in section 侎. It is worthwhile to emphasise how our approach differs from the usual treatment of a system of indistinguishable bosons in quantum mechanics. In the latter context, the $N$-particle sector of the Fock space is constructed as the $N$ th symmetric power of the Hilbert space of a single particle. In our situation, the $N$-particle sector is constructed directly from the quantisation of a classical $N$-particle phase space.

\section{Switching on the potential}

In the self-dual limit $\lambda \rightarrow 1$, the classical hamiltonian (2.15i) is equal to the constant $N \pi$; then, the quantum Hilbert space $\mathcal{H}_{\mathcal{P}}$ is totally degenerate in energy. In the regime 
where $\lambda$ is different but still close to 1 , the vortices move slowly, and we might expect to study their quantum dynamics. For example, one might hope to be able to diagonalise the quantum operator ${ }^{2} \mathcal{Q}\left(V^{\text {red }}\right)$ and in particular determine the ground state of the system. (Notice that when $\lambda \neq 1$ the classical dynamics and its quantisation are still defined by the same symplectic Kähler structure as in the self-dual case.) Unfortunately, any study of the dynamics involves computing matrix elements for the hamiltonian as integrals over the moduli space (cf. ( (i). $\left.\overline{4}^{\prime}\right)$ ), and they cannot be found unless the interaction part $\mathcal{B}$ of the Kähler potential is known to high precision. Nevertheless, physical arguments can be used to give partial answers to these questions.

Although the wavefunctions $\Psi$ in the problem may seem rather abstract, there are two ways to have immediate intuition about them. One is to consider their vanishing loci, which do not depend on the trivialisation of the prequantum line bundle; we can regard them as hypersurfaces of $\mathcal{M}_{N}$ avoided by the configuration of $N$ vortices in the particular state. The other handle is provided by the explicit representation of the rotation group obtained in section configurations symmetric about a given point of the sphere.

Classically, one vortex has intrinsic angular momentum of magnitude $\frac{\kappa}{2}\left(R^{2}-1\right)$. Its geometric quantisation is equivalent to that of a spin state (a classic textbook example, cf. [2]2]), and one can recover the phase space $\mathcal{M}_{1}$ as the Riemann sphere of coherent states. The Hilbert space is still degenerate in energy. For multivortices, the picture is of course more complicated. The interactions built into the symplectic structure imply that nonlinear superposition effects are present. In particular, the total angular momentum of $N$ vortices is not just the sum of the individual angular momenta. But again the coherent states corresponding to the largest multiplet may be interpreted as forming the Riemann sphere $\mathbb{C P}^{1} \cong \mathcal{M}_{N}^{\text {co }} \subset \mathcal{M}_{N}$ of coincident $N$-vortices. The nontriviality of the hamiltonian lifts the energy degeneracy of the self-dual case and in general energy gaps will be formed. We may now ask how this splitting occurs, and in particular which states span the lowest energy level.

The sign of $\lambda-1$ determines whether the potential among the vortices decreases $(\lambda<1)$ or increases $(\lambda>1)$ when a number of separated vortices approach a clustering configuration; we base this presumption on the numerical study of the potential for 2vortices on the plane [2] In the $\lambda<1$ case, the states of lowest energy should correspond classically to coincident vortices, and as stated above they span the largest $S O(3)$ multiplet in $\mathcal{H}_{\mathcal{P}}$. For the $\lambda>1$ case, the problem is more interesting. We expect to find the ground state among the smallest multiplets; roughly speaking, these are states with small angular momentum, which typically correspond to classical configurations of well-spaced vortices.

A way to produce a state with these properties is to enforce vanishing of the wavefunction whenever any two vortices approach each other. The simplest possibility is to

\footnotetext{
${ }^{2}$ See the footnote in section
} 
consider

$$
\Psi_{N, m}^{(0)}:=\prod_{1 \leq r<s \leq N}\left(z_{r}-z_{s}\right)^{m}
$$

We take $m$ to be an even integer, ensuring that this is still a symmetric polynomial in the $z_{r}$, and so of the form (15.5). For this, we must have

$$
m \leq \frac{\ell}{N-1}
$$

because the degree of $\left({ }^{6} \bar{l}_{-}^{\prime}\right)$ in the $s_{k}:=s_{k}^{[N]}\left(z_{1}, \ldots, z_{N}\right)$ is $m(N-1)$. To see this, notice that each $s_{k}$ is if degree 1 in $z_{1}$ say, and $\Psi_{N, m}^{(0)}$ is of degree $m(N-1)$ in $z_{1}$, so each $s_{k^{-}}$ monomial can be of degree at most $m(N-1)$, and there are monomials of degree $m(N-1)$ coming from the monomial of highest degree in $z_{1}$ - this follows from a straightforward induction argument. For example, for $m=2$ and $N=3$ we have

$$
\Psi_{3,2}^{(0)}=-4 s_{2}^{3}-27 s_{3}^{2}+s_{1}^{2} s_{2}^{2}-4 s_{1}^{3} s_{3}+18 s_{1} s_{2} s_{3},
$$

of degree $4=2(3-1)$. Notice that $(\underline{6} .2 \overline{2})$ implies that $\Psi_{N, m}^{(0)}$ can exist only on a sphere of sufficiently large radius,

$$
R \geq \sqrt{N+\frac{m(N-1)}{|\kappa|}} .
$$

Since $\Psi_{N, m}^{(0)}$ is homogeneous in the $z_{r}$, it is an eigenvector of $\mathcal{Q}\left(M_{3}\right)$. One has

$$
\mathcal{Q}\left(M_{3}\right) \Psi_{N, m}^{(0)}=\frac{N}{2}(m(N-1)-\ell) \Psi_{N, m}^{(0)} .
$$

We want to ensure that $\Psi_{N, m}^{(0)}$ has the smallest possible momentum, so it is natural to restrict ourselves to the situation where $R$ is tuned so that $(6.3 .3)$ becomes an equality,

$$
\ell=m(N-1)
$$

Then $\Psi_{N, m}^{(0)}$ has zero momentum about the $x_{3}$-axis. In fact, a tedious calculation that we will not reproduce here establishes that under the condition $\left(\overline{6}^{-} \cdot 4^{\prime}\right)$ also

$$
\mathcal{Q}\left(M_{1}\right) \Psi_{N, m}^{(0)}=0=\mathcal{Q}\left(M_{2}\right) \Psi_{N, m}^{(0)},
$$

using $\left(\overline{5}^{5} \cdot \overline{6}\right)$ and $(\overline{5} . \overline{3})$. Hence the state represented by $\Psi_{N, m}^{(0)}$ is $S O(3)$-invariant, and so a good candidate for the ground state when $\lambda>1$, provided (16.4is) holds.

\section{Discussion}

We have investigated an effective quantisation of Manton's model of first-order vortex dynamics on a sphere of radius $R$. The motion in the classical field theory is first reduced to 
a hamiltonian system on the moduli space of $N$ Bogomol'nyı̆ vortices. At the self-duality point $\lambda=1$, the effective dynamics is frozen, whereas for $\lambda \simeq 1$ the vortices move slowly, preserving their energy and angular momentum; the energy is purely potential and depends on the relative position of the vortices only ${ }^{3}$. The geometric quantisation of the reduced model is then rather straightforward to set up. The prequantum line bundle is uniquely determined by the Kähler structure on the moduli space defined by the kinetic energy term, and it comes with a unique holomorphic structure defined by the natural Kähler polarisation. It is presumed that the quantum system obtained in this way describes a finite truncation of the quantum field theory, in which most of the excitations are kept in the ground state. However, it is not clear how one should assess the validity of this approximation. For $\lambda=1$, the quantisation of the reduced system yields a single degenerate energy level; this degeneracy is lifted when the potential becomes nontrivial. In section we have computed the dimension of the quantum Hilbert space and it was shown that it approaches Gibbs' estimate for the number of quantum states, as determined by the symplectic volume of the moduli space, in the classical limit of large Chern-Simons coefficient. Another feature which emerges from the analysis of the quantised effective system is that the solitons in the model should be interpreted as interacting bosons with a characteristic size of $4 \pi$. The bosonic character of the vortices is also apparent from the analysis of the representations of $S O(3)$ arising in the algebra of the quantum angular momentum operators. In section 15 , we found that for $N$ vortices the Hilbert space is the $N$ th symmetric power of an irreducible representation of $S O(3)$. This irreducible representation is the same as the one obtained from quantising a single vortex on a sphere whose area is the one of the original sphere minus the total area occupied by $N$ vortices.

An interesting aspect of Manton's model is that the classical dynamics of three vortices is integrable in the sense of Liouville, if $\Sigma$ is maximally symmetric (i.e. the sphere, the euclidean plane or the hyperbolic plane) [1] $\left.{ }_{1}^{1} \bar{g}\right]$. However, explicit results about the dynamical regime $\lambda \neq 1$ are difficult to obtain, given the nonlocality of the hamiltonian (2.2.15i). At the quantum level, some statements about the ground state can be made. In particular, we argued in section $\overline{6}_{1}^{\overline{6}}$ that for $\lambda>1$ we expect the ground state to be described by $\left(\overline{6}_{2} . \overline{1}\right)$, which is reminiscent of the Laughlin wavefunction [i] $\left.{ }_{1}^{1}\right]$ in the microscopic approach to the fractional quantum Hall effect (FQHE) [20 $\left.\overline{2}_{3}\right]$. In fact, the connection of our work to the theory of the fractional quantum Hall fluid can be made more explicit. It can be shown that Manton's model is (at least classically) equivalent to the effective field theory for the

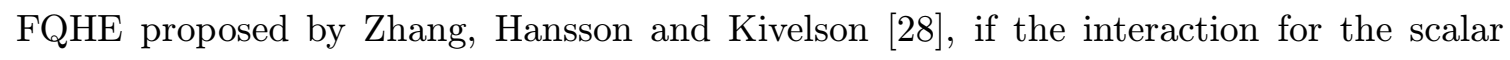
field is set to a $\delta$-function and after suitable rescalings [1] $\left.{ }_{1}^{1} \overline{9}\right]$. From this point of view, the parameter $\gamma$ in $(\overline{2} .8 ;)$ is to be regarded as the electron density. The vortices in this effective field theory have been interpreted [1] model excitations above a basic Laughlin ground state of the electron system. To explain why the FQHE occurs at filling factors not related to a Laughlin wavefunction, Haldane [8] devised a hierarchical scheme whereby the quasiparticles are assumed to behave as

\footnotetext{
${ }^{3}$ It is worth mentioning that the adequacy of the adiabatic approximation to a first-order lagrangian has not yet been studied in detail; we have some indications that this is a reasonable assumption, but a rigorous study in the line of [26] and [20
} 
bosons and can occupy Laughlin-like states $(\overline{6}, \overline{1} \cdot \overline{1})$ that are favoured for certain values of the physical parameters. We believe that our results give some support to Haldane's more heuristic arguments.

\section{Acknowledgments}

The idea of studying the Kähler quantisation of Manton's model was first suggested by G.W. Gibbons. I am grateful to N.S. Manton for many helpful discussions. I would also like to thank the organisers of the IFT/UNESP Workshop on Integrable Field Theories, Solitons and Duality for the opportunity to present this work to an interested audience. This work was financially supported by Fundação para a Ciência e a Tecnologia, Portugal, under the grant $\mathrm{BD} / 15030 / 98$.

\section{References}

[1] Bogomol'nY̌̆, E.B.: The stability of classical solutions. Sov. J. Nucl. Phys. 24, 449-454 (1976)

[2] Bradlow, S.: Vortices in holomorphic line bundles over closed Kähler manifolds. Commun. Math. Phys. 135, 1-17 (1990)

[3] Dunne, G.: Self-dual Chern-Simons theories. Springer-Verlag, 1995

[4] García-Prada, O.: Vortices on Riemann surfaces. in: Coen, S. (Ed.) Seminari di Geometria. 1994-1995. Univ. Stud. Bologna, 1996 (pp. 141-152)

[5] Gibbons, G.W., Manton, N.S.: Classical and quantum dynamics of BPS monopoles. Nucl. Phys. B 274, 183-224 (1986)

[6] Griffiths, P., Harris, J.: Principles of algebraic geometry. John Wiley and Sons, 1978

[7] Guillemin, V., Sternberg, S.: Symplectic techniques in physics. Cambridge University Press, 1984

[8] Haldane, F.D.M.: Fractional quantization of the Hall effect: A hierarchy of incompressible quantum fluid states. Phys. Rev. Lett. 51, 605-608 (1983)

[9] Hartshorne, R.: Algebraic geometry. Springer-Verlag, 1977

[10] Jaffe, A., Taubes, C.: Vortices and monopoles. Birkhäuser, 1980

[11] Laughlin, R.B.: Anomalous quantum Hall effect: An incompressible quantum fluid with fractionally charged excitations. Phys. Rev. Lett. 50, 1395-1398 (1983)

[12] LeE, D.-H.., Zhang, S.-C.: Collective excitations in the Ginzburg-Landau theory of the fractional quantum Hall effect. Phys. Rev. Lett. 66, 1220-1223 (1991)

[13] Manton, N.S.: A remark on the scattering of BPS monopoles. Phys. Lett. B 110, 54-56 (1982)

[14] Manton, N.S.: Statistical mechanics of vortices. Nucl. Phys. B 400 [FS], 624-632 (1993)

[15] Manton, N.S.: First order vortex dynamics. Ann. Phys. 256, 114-131 (1997); hep-th/9701027 
[16] Manton, N.S., Nasir, S.M.: Volume of vortex moduli spaces. Commun. Math. Phys. 199, 591-604 (1999); hep-th/9807017

[17] Manton, N.S., NAsiR, S.M.: Conservation laws in a first-order dynamical system of vortices. Nonlinearity 12, 851-865 (1999); hep-th/9809071

[18] Romão, N.M.: Quantum Chern-Simons vortices on a sphere. J. Math. Phys. 42, 3445-3469 (2001), hep-th/0010277

[19] Romão, N.M.: Classical and quantum aspects of topological solitons. PhD thesis, University of Cambridge, 2002 (unpublished)

[20] Samols, T.M.: Vortex scattering. Commun. Math. Phys. 145, 149-180 (1992)

[21] SнAн, P.A.: Scattering of vortices at near-critical coupling. Nucl. Phys. B 249, 259-276 (1994), hep-th/9402075

[22] ŚNiATYCKI, J.: Geometric quantization and quantum mechanics. Springer-Verlag, 1980

[23] Stone, M. (Ed.): Quantum Hall effect. World Scientific, 1992

[24] Strachan, I.A.B.: Low-velocity scattering of vortices in a modified Abelian Higgs model. J. Math. Phys. 33, 102-110 (1992)

[25] Stunt, D.M.A.: The geodesic approximation for the Yang-Mills-Higgs equations. Commun. Math. Phys. 166, 149-190 (1994)

[26] Stuart, D.: Dynamics of Abelian Higgs vortices in the near Bogomolny regime. Commun. Math. Phys. 159, 51-91 (1994)

[27] Woodhouse, N.: Geometric quantization (2nd edition). Oxford University Press, 1992

[28] Zhang, S.C., Hansson, T.H., Kivelson, S.: Effective-field-theory model for the fractional quantum Hall effect. Phys. Rev. Lett. 62, 82-85 (1989) 